\title{
Design of a high speed and low latency crypto-processor ASIC
}

\begin{abstract}
This paper presents the design of an ultra high speed crypto-processor for next generation IT security. It addresses the next generation IT security requirements: the resistance against all attacks and high speed with low latency. The proposed processor is capable of generating cryptographically secured information at a rate of multi-ten Gbps. The performance of the processor is compared with that of other researchers which proves it's superiority over them.
\end{abstract}

